derived parameters of LA function (LA SV and LA EF) in predicting LV function.

Methods Twelve-patients underwent CMR at 3T (Achieva CV, Philips Healthcare, Best, The Netherlands) within 3 days following AMI. CMR protocol included: cines and late gadolinium enhancement (LGE) imaging ( $0.1 \mathrm{mmol} / \mathrm{kg}$ gadolinium DTPA). Indexed LAEDV (LAEDVi), end-systolic volume (LAESVi) and ejection fraction were computed by bi-plane method. Voxel FT for the LA in the long axis 4-chamber cines was analysed offline using commercially available software (cvi42 v5.1, Circle Cardiovascular Imaging Inc., Calgary, Canada).

Results Demographics and basic CMR parameters are mentioned in Table 1. Analysis time was longer for manual contouring and computation of LA EF and SV compared to 4-CH strain analysis $(4 \pm 2 \mathrm{~min}$ versus $2 \pm 1.5 \mathrm{~min}, \mathrm{p}=0.01)$. On univariate analysis, LV EF was correlated to peak longitudinal strain (PLS) $(\mathrm{p}=0.01)$ and peak radial strain (PRS) $(\mathrm{p}=0.02)$. On multivariate regression analysis, PLS of LA was most strongly correlated to LV EF $(\mathrm{R}=0.68 ; \mathrm{p}=0.015)$. All other parameters did not achieve statistical significance.

Conclusion Peak left atrial longitudinal strain (PLS) is the only parameter of LA function, which independently correlates to LV ejection fraction. PLS of the LA is easily assessed on 4-chamber cines alone and takes less time to compute than the manually generated parameters of LA function.

\begin{tabular}{ll} 
Abstract 14 Table 1 & Demographics and basic CMR values \\
\hline & $\mathrm{n}=\mathbf{1 2}$ \\
\hline Age (years) & $55.4 \pm 11.2$ \\
Male & $75 \%$ \\
Hypertension & $25 \%$ \\
Hypercholesterolaemia & $42 \%$ \\
Smoker & $50 \%$ \\
LV EF & $48 \pm 10$ \\
Infarct Volume (ml) & $16.7 \pm 11.9$ \\
\hline Values expressed as $\mathrm{n}(\%)$ or mean \pm SD
\end{tabular}

\section{PREDICTORS OF RIGHT VENTRICULAR REMODELLING IN REPERFUSED INFERIOR MYOCARDIAL INFARCTIONS: CMR VOXEL FEATURE TRACKING BASED FEASIBILITY STUDY}

P Garg, A Kidambi, DP Ripley, LE Dobson, PP Swoboda, TA Musa, AK McDiarmid, B Erhayiem, P Haaf, JP Greenwood, S Plein. LICAMM, University of Leeds, Leeds, UK

\subsection{6/heartjn|-2015-307845.15}

Background RV function after STEMI has important prognostic implications. However, changes in RV function over time after inferior-STEMI and the incidence of RV remodelling remain unclear. We aimed to investigate which parameters of RV function after inferior-STEMI influence RV remodelling at 3 months. Methods Twenty-one patients underwent CMR at 3T (Achieva CV, Philips Healthcare, Best, The Netherlands) within 3-days and 90-days following reperfused inferior/posterior STEMI. The CMR protocol included: cines and LGE imaging $(0.1 \mathrm{mmol} / \mathrm{kg}$ gadolinium DTPA). Infarct location was determined from LGE images. Indexed RV end-diastolic volume (RVEDVi), end-systolic volume (RVESVi) and ejection fraction were derived from the short-axis stack cines for day-3 and day-90 scans. Offline strain analysis was performed for day-3 scans by voxel feature tracking (FT) for the RV and RA in the 4-chamber cines using commercially available software (cvi42 v5.1, Circle Cardiovascular Imaging Inc., Calgary, Canada).

Results Mean age of our population was $57 \pm 12$ years-old. $86 \%$ patients were male. RV EF improved significantly from day-3 scan to day-90 scans $(40 \pm 12.6 \%$ vs. $49 \pm 10.9 \%$, $\mathrm{p}<0.001)$. Day-3 RV EF demonstrated correlation to RV PLS $(p=0.03)$, RV PRS ( $p=0.03$ ) and RA TTP LSR (0.018). On multivariate stepwise analysis, RV PLS showed the strongest correlation $(\mathrm{R}=0.44 ; \mathrm{p}=0.04)$. Day-90 RV EF was most strongly correlated to TTP of LSR of RA $(R=0.48, p=0.048)$. Relative change in RV EF was also correlated to RV PLS $(\mathrm{p}=0.03)$.

Conclusion Voxel FT derived RV functional parameters, mainly PLS, correlates well with Day $3 \mathrm{RV}$ EF and with relative change of RV EF at day-90. Interestingly, day-90 RV EF showed the strongest correlation to time to peak longitudinal strain rate (TTP LSR) of the RA. This may be because TTP SR parameters reflect mechanical dyssynchrony after the acute ischaemic event. This concept needs further clarification in larger studies.

\section{RELATIONSHIP OF MITRAL ANNULAR PLANE SYSTOLIC EXCURSION AND INTRA-MYOCARDIAL HAEMORRHAGE IN REPERFUSED ST-ELEVATION MYOCARDIAL INFARCTION}

P Garg, A Kidambi, DP Ripley, LE Dobson, PP Swoboda, TA Musa, AK McDiarmid, B Erhayiem, JP Greenwood, S Plein. LICAMM, University of Leeds, Leeds, UK

\subsection{6/heartjnl-2015-307845.16}

Background Mitral annular plane systolic excursion (MAPSE) is known to have prognostic importance in patients with acute myocardial infarction (MI). In post-MI patients with MAPSE $<8 \mathrm{~mm}$, the combined mortality and hospitalisation incidence is $43.8 \%$. Similarly, intra-myocardial haemorrhage (IMH) in the infarct-core is an independent marker of prognosis. We hypothesised that the MAPSE on 4-chamber cine-CMR is correlated to left ventricular ejection fraction $(\mathrm{EF})$ and to the presence of IMH.

Methods Fourty-four patients received CMR examination at 3T (Achieva CV, Philips Healthcare, Best, The Netherlands) within 3 days following acute MI. Cine, T2-weighted, T2*-imaging and late gadolinium enhancement (LGE) imaging were performed. Infarct and microvascular obstruction (MO) extent were measured from LGE images. The presence and extent of IMH was investigated by combined analysis of $\mathrm{T} 2 \mathrm{w}$ and $\mathrm{T} 2 *$ sequences. MAPSE was computed (medial, lateral and average) using the 4-chamber cine (Figure 1).

Results Mean age of our studied population was 58.27 \pm 11.41. CMR parameters were as follows: LVEF $48.2 \pm 11.4 \%$; infarct volume of $15.5 \pm 12.2 \mathrm{ml}$ and averaged MAPSE of $10.27 \pm 2.1 \mathrm{~mm}$. Controlling for risk factors, IMH was strongly negatively correlated to average MAPSE $(\mathrm{r}=-0.65$; $\mathrm{p}<0.001)$. Averaged MAPSE was also moderately correlated to LVEF $(\mathrm{r}=0.47 ; \mathrm{p}=0.001)$.

Conclusion Averaged MAPSE, which is a simple CMR derived parameter of longitudinal function, has the potential to predict the presence of IMH in the setting of re-perfused acute MI. This parameter could be easily measured at bedside by transthoracic echocardiography to predict presence of IMH.

Funding JPG and SP receive a research grant from Philips Healthcare. SP is funded by British Heart Foundation fellowship (FS/10/62/28409). 


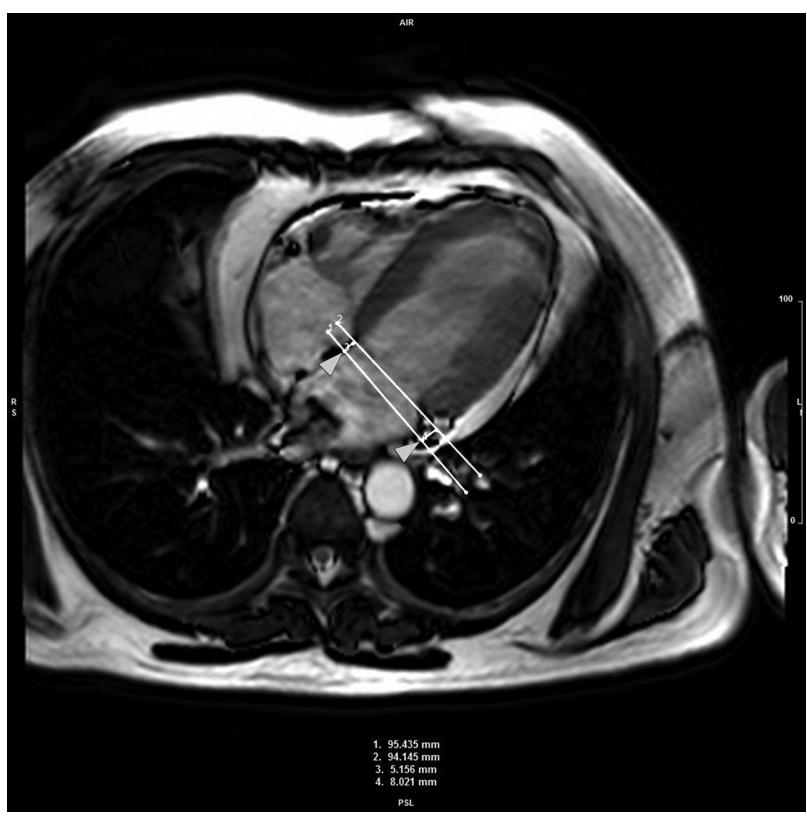

Abstract 16 Figure 1 MAPSE measurements were taken in the fourchamber cine.

\section{ROUTINE CARDIAC MRI SCREENING REVEALS REDUCED PREVALENCE OF STRUCTURAL CARDIOVASCULAR ABNORMALITIES IN PATIENTS WITH TURNER SYNDROME}

\begin{abstract}
${ }^{1}$ Agnes Hamilton-Baillie, ${ }^{1,2}$ Christopher M Jones, ${ }^{1}$ Caroline Packer, ${ }^{2}$ Andy Toogood, ${ }^{2}$ Paul Clift. 'College of Medical and Dental Sciences, University of Birmingham, UK; ${ }^{2}$ University Hospitals Birmingham NHS Foundation Trust, Birmingham, UK
\end{abstract}

\subsection{6/heartjnl-2015-307845.17}

Background Turner syndrome has been associated with significant rates of cardiovascular anomalies. Current guidelines for the management of this condition suggest evaluation of the aorta via cardiac magnetic resonance (CMR) imaging or echocardiography every 5 to 10 years to evaluate each patient's potential for aortic dissection, without prior risk stratification. The current spectrum and frequency of structural cardiovascular anomalies in Turner syndrome is however unclear, with much of the current literature based on historical cohorts.

Purpose To determine through CMR screening the current prevalence of structural cardiovascular anomalies amongst patients with Turner syndrome.

Methods We retrospectively reviewed the medical records of adult patients diagnosed with Turner syndrome who had been seen within a large NHS tertiary referral centre and who had undergone routine surveillance for structural cardiovascular anomalies. Each underwent CMR imaging. Data relating to patient demographics and baseline observations were extracted. The presence of structural cardiac anomalies was determined through analysis of MRI and echocardiogram imaging, in addition to review of records relating to previous operative intervention.

Results Medical records for 46 patients with Turner syndrome were reviewed, 7 (15.2\%) of whom were mosaic for $\mathrm{X}$-chromosomal monosomy. Median age was 28 years (range 21-73 years). 19/46 (41.3\%) patients had no reported structural cardiovascular anomaly. Of the 27 patients with structural anomalies, $19(70.4 \%)$ were reported to feature a bicuspid aorta, $13(28.3 \%)$ coarctation of the aorta and $10(21.7 \%)$ dilated aortic root. 13/46 (28.3\%) patients underwent surgical intervention, including 11 (23.9\%) who underwent repair of aortic coarctation.

Conclusions The presence of cardiovascular abnormalities amongst our population was lower than that reported within the literature. Patients with Turner syndrome are nevertheless likely to undergo surgical intervention. Risk stratification tools are therefore required to optimise use of resources in patients with Turner syndrome requiring routine screening for structural cardiac anomalies.

Conflicts of interest The authors declare that they have no conflicts of interest relating to this article.

\section{RELATIONSHIP OF MYOCARDIAL STRAIN AND MARKERS OF MYOCARDIAL INJURY TO PREDICT SEGMENTAL RECOVERY FOLLOWING ACUTE ST-SEGMENT ELEVATION MYOCARDIAL INFARCTION}

${ }^{1} \mathrm{JN}$ Khan*, ${ }^{2} \mathrm{JP}$ Greenwood, 'SA Nazir, ${ }^{1} \mathrm{~A}$ Singh, ${ }^{3} \mathrm{C}$ Peebles, ${ }^{4} \mathrm{~J}$ Wong, ${ }^{1} \mathrm{AH}$ Gershlick, ${ }^{1}$ GP McCann. 'Department of Cardiovascular Sciences, University of Leicester and the NIHR Leicester Cardiovascular Biomedical Research Unit, University Hospitals of Leicester NHS Trust, Glenfield Hospital, Leicester, LE3 9QP, UK; ${ }^{2}$ Multidisciplinary Cardiovascular Research Centre and the Division of Cardiovascular and Diabetes Research, Leeds Institute of Cardiovascular and Metabolic Medicine (LICAMM), University of Leeds, Leeds, LS2 9JT, UK; ${ }^{3}$ University Hospital Southampton NHS Foundation Trust and University of Southampton, Southampton SO16 6YD, UK; ${ }^{4}$ Royal Brompton and Harefield Foundation Trust, Harefield Hospital, Hill End Road, Middlesex UB9 6JH, UK

\subsection{6/heartjnl-2015-307845.18}

Background Late Gadolinium Enhancement (LGE) predicts functional recovery in stunned myocardium. Acutely post STEMI, LGE overestimates infarct and underestimates potential for functional recovery. There are no large studies comparing CMR predictors of segmental recovery in acute STEMI.

Purpose Determine whether segmental circumferential strain (Ecc), myocardial salvage (MSI), microvascular obstruction (MVO) and intramyocardial haemorrhage (IMH) predict segmental functional recovery and offer incremental predictive value to segmental extent of enhancement (SEE) acutely postPPCI.

Methods 1.5T CMR was performed in 164 patients (2624 segments) at $48 \mathrm{hr}$ and 9 month post-STEMI. LV function was assessed on wall-motion scoring on SSFP cines. Segmental dysfunction was WMS $\geq 2$, improvement was WMS decrease of $\geq 1$ and normalisation where WMS returned to 1 at follow-up. SEE and MVO were assessed on LGE using full-width half-maximum thresholding. Oedema and IMH were assessed on T2w imaging using Otsu's Automated Method. Ecc was Feature Trackingderived. MSI was the proportion of non-infarcted segmental oedema. Accuracy of baseline SEE, segmental Ecc, MSI, MVO and $\mathrm{IMH}$ in predicting improvement and normalisation in dysfunctional segments was assessed with Receiver Operator Curves.

Results 32\% of segments were dysfunctional at baseline and $19 \%$ at follow-up. With increasing SEE, segmental function worsened and proportion of dysfunctional segments recovering decreased. However $33 \%$ of SEE $>75 \%$ segments improved (Figure 1a). SEE was a strong predictor of improvement (AUC 0.708 ) and normalisation (AUC 0.807). SEE was a stronger predictor than MVO, IMH and Ecc ( $p<0.01$ for all). MVO, IMH and Ecc were weak predictors. Combining SEE with MVO, 